\title{
Cold, dilute, trapped bosons as an open quantum system
}

\author{
James Anglint \\ Theoretical Astrophysics, MS B288, Los Alamos National Laboratory, Los Alamos, New Mexico 87545
}

We present a master equation governing the reduced density operator for a single trapped mode of a cold, dilute, weakly interacting Bose gas; and we obtain an operator fluctuationdissipation relation in which the Ginzburg-Landau effective potential plays a physically transparent role. We also identify a decoherence effect that tends to preserve symmetry, even when the effective potential has a "Mexican hat" form.

PACS numbers: 03.75.Fi, 05.30.Jp, 11.30.Qc, 34.40.+n

The recent observations of Bose condensation in magnetically trapped gases 2, 3, 4, ,5, 6, have brought phenomena hitherto reserved to condensed matter physics into the interdisciplinary field now growing between quantum optics and mesoscopic physics. In particular, this breakthrough may offer a weakly interacting system in which to study one of the most interesting of quantum phenomena: the spontaneous breaking of number eigenstates, which are invariant (up to a Hilbert space phase) under the rotation $\hat{U}=e^{i \theta \hat{n}}$, into coherent states, which transform as $\hat{U}|\alpha\rangle=\left|e^{i \theta} \alpha\right\rangle$. Such symmetry breaking has long been thought to be a basic cause of superfluidity and superconductivity. Yet the breaking of the symmetry whose Noether charge is particle number is still rather more mysterious than the symmetry breaking involved in, say, the Standard Model of particle theory, because instead of being due to an instability in the microscopic Hamiltonian of the system, it is thought to be driven by an effective potential of the form

$$
V=\left(E_{1}-\mu\right)|\psi|^{2}+E_{2}|\psi|^{4}
$$

where $\psi$ is a second-quantized destruction operator.

While it has long been customary to include an effective potential of this form in the Hamiltonian for a gas of weakly interacting bosons [7], the presence of the chemical potential $\mu$ must remind us that this effective potential (EP) is fundamentally a property of the system's thermal environment. In non-equilibrium dynamics, therefore, the EP should most properly appear, like temperature, in the fluctuation-dissipation relation (FDR). To realize such a result, we must consider Bose condensation, and the possibly concomitant symmetry breaking, to occur in an open quantum system. The open system paradigm is not only natural for gases that are cooled by evaporation and thereafter steadily leak out of their traps; it is in general a powerful lingua franca for describing the rapidly growing common ground between quantum optics, mesoscopic quantum mechanics, and condensed matter physics. Recent work using this paradigm has developed analogs of laser theory for atoms [8.9.10] and excitons [11]. The nonlinearity of the interaction between hard spheres makes open system calculations difficult for gases in general, but in this Letter we present an idealized model of a cold, dilute, trapped Bose gas, in which the problem may be solved analytically to leading order in small parameters. We are thereby able to obtain the fully nonlinear, fully quantum mechanical FDR, and so make contact between the open system approach and traditional many-body formulations based on an EP.

Instead of the quadratic confining potentials of real experiments, we imagine a Bose gas subjected to a deep but narrow spherical square potential well, tuned so as to possess exactly one single-particle bound state. In the second-quantized formalism, this single-particle state becomes a normal mode, with a discrete set of energy levels. We treat this mode as an open quantum system, interacting, via a short-ranged two-particle potential, with a reservoir consisting of the continuum of unbound modes. We then outline a derivation (to be presented in detail elsewhere) that uses Feynman's ordered operator calculus 12] to obtain a Markovian master equation for the reduced density operator of the bound mode. This equation contains condensate growth and depletion terms whose relative strength is characterized by an operator fluctuation-dissipation relation, which is physically transparent, and in which the Ginzburg-Landau EP may be discerned. We also find an additional phase diffusion term, representing quantum decoherence due to scattering of unbound particles by the condensate. We will discuss the implications of this term for symmetry breaking at the end of this Letter.

We therefore begin with the second-quantized Hamiltonian

$$
\begin{gathered}
\hat{H}=\frac{\hbar^{2}}{2 m} \int d V\left[\vec{\nabla} \hat{\psi}^{\dagger} \cdot \vec{\nabla} \hat{\psi}-2 \lambda^{2} \theta(\pi-\lambda r) \hat{\psi}^{\dagger} \hat{\psi}\right. \\
\left.+4 \pi a \hat{\psi}^{\dagger} \hat{\psi}^{\dagger} \hat{\psi} \hat{\psi}\right],
\end{gathered}
$$

where $\theta$ is the step function, and $a$ is the scattering length of the gas particles $\left(\sigma=16 \pi a^{2}\right.$ being the cross section for two hard-sphere bosons scattering with momentum transfer small compared to $\left.a^{-1}\right)$. We then diagonalize the quadratic part of $\hat{H}$, by defining $\hat{\psi}(\vec{r})=$ $\sum_{k l m} \hat{\psi}_{k l m} u_{k l m}(\vec{r})$, such that

$$
\left(\nabla^{2}+k^{2}\right) u_{k l m}=2 \lambda^{2} \theta(\pi-\lambda r) u_{k l m} .
$$


The $u_{k l m}$ with real $k$ are unbound modes, scattered by the well 13. We will let the spectrum of $k$ approach a continuum by implicitly assuming a boundary condition at large radius. There is also exactly one bound solution, which is spherically symmetric; by solving a transcendental equation we can compute its binding energy to be $E_{b} \doteq 0.457 \frac{\hbar^{2} \lambda^{2}}{2 m}$. We denote this bound mode wave function by $u_{B}(\vec{r})$, and its creation and destruction operators by $\hat{\psi}_{B}^{\dagger}$ and $\hat{\psi}_{B}$. The bound mode will then constitute the observed system, and the continuum modes the environmental reservoir, which will be in self-equilibrium with temperature $\left(k_{B} \beta\right)^{-1}$ and chemical potential $\mu$, though it may be far from equilibrium with the bound mode.

We then re-write the Hamiltonian (2) in terms of the normal modes, splitting it into bound mode, continuum, and interaction parts:

$$
\begin{aligned}
\hat{H} & =\hat{H}_{B}+\hat{H}_{C}+\hat{H}_{I} \\
\hat{H}_{B} & =-E_{b} \hat{n}_{B}+E_{r} \hat{n}_{B}\left(\hat{n}_{B}-1\right) \\
\hat{H}_{C} & =\frac{\hbar^{2}}{2 m} \sum_{k l m} k^{2} \hat{\psi}_{k l m}^{\dagger} \hat{\psi}_{k l m}+\hat{H}_{s c a t} \equiv \hat{H}_{k i n}+\hat{H}_{\text {scat }},
\end{aligned}
$$

where $\hat{n}_{B} \equiv \hat{\psi}_{B}^{\dagger} \hat{\psi}_{B}$, and the condensate self-repulsion energy $E_{r}$ is of order $a \hbar^{2} \lambda^{3} m^{-1}$, proportional to $\int d^{3} r u_{B}^{4}$. $\hat{H}_{\text {scat }}$ is a quartic operator which produces two-particle scattering among the continuum modes. It will be convenient to use the interaction picture, in which operators evolve under $\hat{H}_{B}$ and $\hat{H}_{C}$ :

$$
\begin{aligned}
\hat{\psi}_{B}(t) & =e^{\frac{i}{\hbar}\left(E_{b}-2 E_{r} \hat{n}_{B}\right) t} \hat{\psi}_{B}(0) \equiv e^{-i \hat{\Delta} t} \hat{\psi}_{B}(0) \\
\hat{\psi}_{k l m}(t) & =e^{-i \frac{\hbar k^{2}}{2 m} t} \hat{\Psi}_{k l m}(t) .
\end{aligned}
$$

Note that $\hat{\Delta}=\Delta(\hat{n})$ is simply the frequency difference between the $n$th and the $(n-1)$ th eigenstate of $\hat{H}_{B}$. The operators $\hat{\Psi}_{k l m}(t)$ evolve under $\hat{H}_{\text {scat }}$. This evolution is complicated, but for our purposes it turns out to be sufficient to note that it makes the two-point function decay with time difference. In the case where the gas is dilute, we can take sufficient account of this effect by approximating

$$
\operatorname{Tr}\left[\hat{\rho}(\beta, \mu) \hat{\Psi}_{k l m}(t) \hat{\Psi}_{k^{\prime} l^{\prime} m^{\prime}}^{\dagger}\left(t^{\prime}\right)\right] \doteq \delta_{k k^{\prime}} \delta_{l l^{\prime}} \delta_{m m^{\prime}} e^{-\gamma_{k}\left|t-t^{\prime}\right|},
$$

where $\hat{\rho}(\beta, \mu)$ is the grand canonical ensemble density operator, and $\gamma_{k}=\hbar k \sigma d / m$ is the Boltzmann scattering rate, for $d=e^{\beta \mu}\left(m / 2 \pi \hbar^{2} \beta\right)^{3 / 2}$ the density of the gas [14]. The equilibration time for the unbound gas is set by the thermal average of $\gamma_{k}$, which we will denote by $\gamma$.

In terms of the time-dependent operators, then, we can write the interaction picture $\hat{H}_{I}$ [using the notation $\vec{k}=(k, l, m)]$

$$
\hat{H}_{I}(t)=2 \pi \frac{\hbar^{2} a}{m}\left[\sum_{\vec{k}_{i}}\left[V_{3}\left(\vec{k}_{i}\right) \hat{\psi}_{\vec{k}_{1}}^{\dagger} \hat{\psi}_{B}^{\dagger} \hat{\psi}_{\vec{k}_{2}} \hat{\psi}_{\vec{k}_{3}}+\text { h.c. }\right]\right.
$$

$$
\begin{aligned}
& +\sum_{k k^{\prime} l m}\left[V _ { 2 } ( k , k ^ { \prime } , l ) \left(\hat{\psi}_{k l m}^{\dagger} \hat{\psi}_{k^{\prime} l,-m}^{\dagger} \hat{\psi}_{B} \hat{\psi}_{B}\right.\right. \\
& \left.\left.+2 \hat{\psi}_{k l m}^{\dagger} \hat{\psi}_{k^{\prime} l m} \hat{n}_{B}\right)+ \text { h.c. }\right] \\
& \left.+\sum_{k} V_{1}(k) \hat{\psi}_{B}^{\dagger}\left(\hat{\psi}_{B}^{\dagger} \hat{\psi}_{k 00}+\hat{\psi}_{k 00}^{\dagger} \hat{\psi}_{B}\right) \hat{\psi}_{B}\right]
\end{aligned}
$$

where all the $V_{n}$ co-efficients are given by integrals of four mode functions $\left(u_{k l m}\right.$ and $\left.u_{B}\right)$.

Our goal is to compute the reduced density operator of the bound mode, from the initial density operator of the bound mode and the reservoir. In the interaction picture, this is given by

$$
\begin{aligned}
& \hat{\rho}_{B}(t)=\operatorname{Tr}_{C} {\left[\mathcal{T} e^{-\frac{i}{\hbar} \int_{0}^{t} d s \hat{H}_{I}(s)}\right.} \\
&\left.\times\left[\hat{\rho}_{B}(0) \otimes \hat{\rho}_{C}(0)\right] \overline{\mathcal{T}} e^{\frac{i}{\hbar} \int_{0}^{t} d s \hat{H}_{I}(s)}\right],
\end{aligned}
$$

where $\mathcal{T}(\overline{\mathcal{T}})$ denote (reverse) time ordering. We have assumed that the initial density matrix factorizes, and we will take $\hat{\rho}_{C}(0)$ to be a grand canonical ensemble $\hat{\rho}(\beta, \mu)$. In much the same spirit as that of the influence functional formalism, we will determine the evolution of the reservoir degrees of freedom and perform the trace over them before considering the bound mode itself. The bound mode operators $\hat{\psi}_{B}(t)$ are not operators in the bath Hilbert space; but we are apparently prevented from treating them merely as c-numbers in the bath Hamiltonian, because they are still operators in the bound mode Hilbert space, and their ordering is significant. Yet as Feynman observed, this difficulty may be overcome by a simple notational trick [12].

In a slight generalization of Feynman's original ordered operator calculus, we here add the device of placing primes on all $\hat{\psi}_{B}(t)$ operators that appear to the right of the initial density operators in Eqn. (8). We will then evaluate the RHS of (8) just as if the bound mode operators were not operators at all, but keep track of their time arguments. We will afterwards be able to restore their correct ordering as operators, simply by placing all unprimed operators time ordered to the left of $\hat{\rho}_{B}(0)$, and all primed operators in reverse time order to the right. This sort of procedure can always be used, of course; but in general it provides only an opaque formal solution in terms of time ordered operators. The convenient features of the present idealized model happen to make it genuinely powerful here.

Performing the standard equilibrium analysis after momentarily setting $a \rightarrow 0$ confirms that if $\beta E_{b}>>1$, Bose condensation occurs at very low fugacity $e^{\beta \mu} \sim e^{-\beta E_{b}}$. Assuming both $\beta$ and $\mu$ to be in this regime, then, we can evaluate (8) in the dilute gas approximation, where we consider only independent two particle collisions. (This approximation affects only the unbound bath modes: we are not hereby making any assumption concerning the bound mode particles, or interactions between them!) 
We therefore replace Eqn. (8) with the dilute gas expression

$$
\begin{aligned}
\hat{\rho}_{B}(t)= & \hat{\rho}_{B}(0) \exp \left[-\frac{i}{\hbar} \int_{0}^{t} d s \operatorname{Tr}\left[\hat{\rho}_{C}(0)\left(\hat{H}_{I}-\hat{H}_{I}^{\prime}\right)\right]\right. \\
& +\frac{1}{2 \hbar^{2}}\left(\int_{0}^{t} d s \operatorname{Tr}\left[\hat{\rho}_{C}(0)\left(\hat{H}_{I}-\hat{H}_{I}^{\prime}\right)\right]\right)^{2} \\
- & \frac{1}{\hbar^{2}} \int_{0}^{t} d s \int_{0}^{s} d s^{\prime} \operatorname{Tr}\left[\hat{\rho}_{C}(0)\left(\hat{H}_{I}-\hat{H}_{I}^{\prime}\right)_{s}\left(\hat{H}_{I}-\hat{H}_{I}^{\prime}\right)_{s^{\prime}}\right],
\end{aligned}
$$

where the RHS is to be evaluated using the ordering convention described above for the bound mode operators.

The second simplification purchased by our idealizations is a time scale separation, which allows us to collapse the double time integrals in (9) into single integrals, and so obtain Markovian evolution for the bound mode. The Boltzman scattering rate is proportional to $e^{\beta \mu}$, but the condensate grows through collisions between two gas particles in the trapping well, which (since the well has a finite volume) occur at a rate proportional to $e^{2 \beta \mu}$. We can therefore use approximations based on the fact that the condensate evolves much more slowly than the reservoir equilibrates. Since there are several different terms in $\hat{H}_{I}$, we must in fact employ several variations of this approximation, and also use the assumption of weak interaction $(\lambda a<<1)$. This analysis will be described in detail elsewhere, but we will illustrate the least trivial and most important step here.

The following important double time integral from (9) represents a depletion event in which an unbound particle of momentum $k_{1}$ dislodges a bound particle, resulting in two unbound particles of momenta $k_{2}$ and $k_{3}$ :

$$
\begin{aligned}
& \int_{0}^{t} d s \int_{0}^{t} d s^{\prime} e^{-\frac{i \hbar}{2 m}\left(s-s^{\prime}\right)\left(k_{1}^{2}-k_{2}^{2}-k_{3}^{2}\right)} e^{-\gamma_{23}\left|s-s^{\prime}\right|} \\
\times & \times \hat{\psi}_{B}(s) \hat{\psi}_{B}^{\dagger^{\prime}}\left(s^{\prime}\right) \\
\doteq & \int_{0}^{t} d s_{+} \hat{\psi}_{B}\left(s_{+}-\epsilon\right) \hat{\psi}_{B}^{\dagger^{\prime}}\left(s_{+}-\epsilon\right) \\
& \times \int_{-s_{+}}^{s_{+}} d s_{-} e^{-\gamma_{23}\left|s_{-}\right|} e^{i s_{-}\left[\frac{\hbar}{2 m}\left(k_{2}^{2}+k_{3}^{2}-k_{1}^{2}\right)-\frac{\hat{\Delta}_{\left(s_{+}\right)+\hat{\Delta}^{\prime}\left(s_{+}\right)}}{2}\right]} \\
= & \mathcal{O}(\gamma)+\pi \int_{0}^{t} d s \hat{\psi}_{B}(s-\epsilon) \hat{\psi}_{B}^{\dagger^{\prime}}(s-\epsilon) \\
& \times \delta\left(\frac{\hbar}{2 m}\left(k_{1}^{2}-k_{2}^{2}-k_{3}^{2}\right)+\frac{\hat{\Delta}(s)+\hat{\Delta}^{\prime}(s)}{2}\right)
\end{aligned}
$$

where $\gamma_{23}$ denotes $\gamma_{k_{2}}+\gamma_{k_{3}}$, and in the last line we have assumed that $t>>\gamma^{-1}$. (Nothing significant happens to the bound mode prior to this, anyway.) The critical step is changing the time arguments of the operators from $s$ and $s^{\prime}$ to $s_{+}=\left(s+s^{\prime}\right) / 2$. This can be done because when the exponentials in (9) are expanded, the typical time between terms like (10) is proportional to $e^{-2 \beta \mu}$, but the difference in each such term between $s$ and $s^{\prime}$ can be only of order $e^{-\beta \mu}$. Our change of time arguments thus only affects the ordering of $\hat{\psi}_{B}$ and $\hat{\psi}_{B}^{\dagger}$ operators at order $e^{3 \beta \mu}$. (Ordering errors involving $\hat{n}_{B}$ operators must also be considered, but this can be shown only to renormalize $\gamma_{23}$.)

The delta function that results from the time scale separation enforces self energy $\left(H_{B}+H_{k i n}\right)$ conservation to leading order in $a^{2}$, and thus realizes the expectation that the dynamics of a dilute gas is dominated by elastic collisions. (In terms from (9) with all operators primed or all unprimed, there is an imaginary part which is not a delta function; but these parts constitute order $a^{2} \lambda^{2} e^{\beta \mu}$ or smaller renormalizations of the bound mode Hamiltonian, negligible in comparison with the bare $\hat{H}_{B}$.) It is crucially important that the delta function we obtain has an operator-valued argument. The legitimacy of this curious expression, despite its somewhat unnerving appearance, shows the power of Feynman's ordered operator calculus, for it implies the correct operator fluctuationdissipation relation between condensate growth and depletion. This result seems to be much more difficult to obtain by other methods.

(It is at this point possible to substantially improve our dilute gas approximation by taking into account depletion of the gas modes during condensation. For brevity, however, we will here assume that the number of condensate particles remains a negligible fraction of the number of bath particles, so that gas depletion is insignificant.)

Having in similar fashion collapsed all the double integrals in (9), we obtain for the bound mode reduced density operator a Markovian master equation of BlochLindblad form. Dropping most of the $B$ subscripts, returning to the Schrödinger picture where $\hat{\rho}$ is the only time-dependent operator, and abandoning the timeordered convention in favour of the ordinary "as-written" ordering, it becomes

$$
\begin{aligned}
& \frac{d \hat{\rho}}{d t}=\frac{i}{\hbar}\left[\hat{\rho}, \hat{H}_{B}\right]-\alpha_{2} \gamma\left(\left\{\hat{n}^{2}, \hat{\rho}\right\}-2 \hat{n} \hat{\rho} \hat{n}\right) \\
&-\alpha_{1} \frac{\gamma e^{\beta \mu}}{\beta E_{b}} \sum_{ \pm}\left(\left\{\hat{Q}_{ \pm}^{\dagger} \hat{Q}_{ \pm}, \hat{\rho}\right\}-2 \hat{Q}_{ \pm} \hat{\rho} \hat{Q}_{ \pm}^{\dagger}\right)
\end{aligned}
$$

where $\alpha_{n}$ are constants of order unity which can be evaluated easily to leading order in $\left(\beta E_{b}\right)^{-1}$, and the condensate feeding and depleting operators $\hat{Q}_{ \pm}$are defined to be

$$
\begin{aligned}
& \hat{Q}_{+} \equiv \hat{\psi}^{\dagger} \\
& \hat{Q}_{-} \equiv e^{\frac{\beta}{2}(\hbar \hat{\Delta}-\mu)} \hat{Q}_{+}^{\dagger} .
\end{aligned}
$$

This master equation is our main result; it is correct to second order in $a \lambda$ and leading order in $e^{\beta \mu}$. While we have made free use of special features of our model to derive it, we conclude by making three observations on the very general physics it represents.

Firstly, we note the terms that do not appear in the master equation. $\hat{H}_{I}$ contains vertices (proportional to 
$V_{2}$ and $V_{3}$ ) which annihilate one or two bound mode particles and create only one or two gas particles (or vice versa); but these vertices do not conserve $H_{B}+H_{k i n}$, and so (apart from some more of the negligible corrections to $\hat{H}_{B}$ mentioned above) can only contribute terms of order $e^{\beta \mu}(a \lambda)^{4}$. Excitation of virtual particles from the condensate may certainly be less negligible in other models; but an energy gap will always tend to suppress such processes in favour of elastic ones.

Secondly, it is easy to show that under (11) $\hat{\rho}$ relaxes towards the grand canonical ensemble $Z^{-1} e^{\beta\left(\mu \hat{n}-\hat{H}_{B}\right)}$, with the full, non-linear $\hat{H}_{B}$. We can consider Eqn. (12) to be an operator FDR which ensures such relaxation. Moreover, it is easy to understand this FDR. As we have noted, the condensate growth process requires two gas particles to collide over the well, and its strength is thus proportional to $e^{2 \beta \mu}$. The depletion process requires only one incident gas particle, and so is of order $e^{\beta \mu}$, but since energy must be conserved to leading order in $a^{2} \lambda^{2}$, the incoming particle must have high energy in order to dislodge a bound particle. Depletion is thus enhanced relative to growth by a (fugacity) ${ }^{-1}$ factor, but suppressed by a thermal factor. (Since the processes involve two $\hat{Q}_{ \pm}$operators, the factors in (12) are the square roots.) And our operator delta function in (10) implies that the thermal factor is precisely $e^{\beta \hbar \hat{\Delta}}$, correctly reflecting the fact that interparticle repulsion lowers the binding energy as $n$ increases. Furthermore, we can note that $\hbar \hat{\Delta}-\mu=V_{G L}(\hat{n})-V_{G L}(\hat{n}-1)$, where

$$
V_{G L}(\hat{n})=\hat{H}-\mu \hat{n}=\left(-E_{b}-\mu\right) \hat{\psi}^{\dagger} \hat{\psi}+E_{r} \hat{\psi}^{\dagger 2} \hat{\psi}^{2}
$$

is the quantized Ginzburg-Landau effective potential. So the Ginzburg-Landau EP simply describes a competition between capture of slow gas particles and ejection by fast particles, both processes proceeding through elastic collisions.

Thirdly, Eqn. (11) also includes the $\alpha_{2}$ term, which is due to gas particles scattering off the bound mode condensate via the elastic part of the $V_{2}$ vertex in $\hat{H}_{I}$. This term represents diffusion in the phase canonically conjugate to particle number, as may be seen by expressing (11) in the Wigner representation, and writing $q+i p \sim \psi=\sqrt{n} e^{i \theta}$. The $\alpha_{2}$ term then becomes a second derivative with respect to $\theta$. While thinking of the real and imaginary parts of $\psi$ as independent classical degrees of freedom may suggest that diffusion in $\theta$ should be of the form $\frac{1}{n} \frac{\partial^{2}}{\partial \theta^{2}}$, in fact the $\alpha_{2}$ term does not decay with $n$ : It does not represent classical noise, but decoherence. Yet the argument that a "Mexican hat" form for $V$ immediately implies spontaneous breaking of rotational symmetry in $\theta$ is actually based on the assumption that, when $n$ is large, diffusion in $\theta$ becomes negligible.

It is certainly possible that decoherence will permit spontaneous symmetry breaking in other models (though perhaps only for the relative phase(s) between two or more condensate modes [15); but scattering of uncondensed particles is a generic process, which may typically be expected to produce phase diffusion terms resembling that obtained here. These terms may well fail in some way to prevent symmetry breaking in other models, but they will not obviously vanish due to mere kinematics as the number of condensed particles becomes large. This strongly suggests the following general conclusion.

Spontaneous symmetry breaking, in which states of definite particle number are replaced by states where the phase which is conjugate to particle number is definite instead, is important in many areas of physics. A "Mexican hat" form of effective potential may be a necessary condition for such SSB, but it is not sufficient, because diffusion associated with decoherence occurs in addition to the diffusion characterized by the effective potential. This implies that emergence of the symmetry breaking order parameter in these cases is not a quasi-classical process, but is an especially non-trivial instance of classicality emerging from quantum mechanics.

\section{Acknowledgements}

The author appreciated the hospitality of the Institute for Theoretical Physics at UCSB, supported by NSF grant PHY94-07194, during the later stages of this work. He thanks A. Gill, E. Mottola, A. Imamoglu, P. Zoller and S. Habib for valuable discussions.

[1] e-mail: anglin@lanl.gov

[2] M.H. Anderson et al., Science 269, 189 (1995).

[3] C.C. Bradley et al., Phys. Rev. Lett. 75, 1687 (1995).

[4] K.B. Davis et al., Phys. Rev. Lett. 75, 3969 (1995).

[5] M.-O. Mewes et al. Phys. Rev. Lett. 77, 416 (1996).

[6] D.S. Din et al. Phys. Rev. Lett. 77, 420 (1996).

[7] See, for instance, A.L. Fetter and J.D. Walecka, Quantum Theory of Many Particle Systems (McGraw-Hill, 1971), p. $488 \mathrm{ff}$.

[8] A.M. Guzmán, M. Moore and P. Meystre, Phys. Rev. A 53, 977 (1995).

[9] H.M. Wiseman and M.J. Collett, Phys. Lett. A 202, 246 (1995).

[10] M. Holland et al., Phys. Rev. A 54, R1757 (1996).

[11] A. Imamoḡlu and R.J. Ram, Phys. Lett. A 214, 193 (1996).

[12] R.P. Feynman, Phys. Rev. 84, 108 (1951).

[13] C. Cohen-Tannoudji et al., Quantum Mechanics (Wiley, 1977), p. 962.

[14] L.P. Kadanoff and G. Baym, Quantum Statistical Mechanics (Benjamin, 1962), Ch. 4.

[15] M. Naraschewski et al., Phys. Rev. A 54, 2185 (1996). 